\title{
Progress towards an Absolute-Scale Quantification of HRTEM Images
}

\author{
A. Thust and J. Barthel \\ Ernst Ruska-Centre for Microscopy and Spectroscopy with Electrons (ER-C) \\ and Institute of Solid State Research, Forschungszentrum Jülich GmbH, D-52425 Jülich, Germany
}

The characterization of the instrumental imaging properties plays a key role in quantitative highresolution transmission electron microscopy (HRTEM). Since the contrast of high-resolution images is determined by the combination of object properties with instrumental contrast-transfer properties, a reliable and quantitative image analysis requires a strict separation of these two different contrast forming mechanisms. The measurement of the instrumental contrast-transfer properties is thus a prerequisite for almost any quantitative HRTEM technique. Knowledge of the instrumental transfer properties is e.g. indispensable for the correct adjustment of hardware aberration correctors, for the successful application of phase retrieval techniques, and for realistic image simulations. We report here on major progress in the three different fields of (i) aberration measurement, (ii) determination of the information limit, and (iii) measurement of the camera modulation-transfer-function.

Concerning aberration measurement, we developed a software suite for ultra-precise aberration measurement, which is in our view a near-optimum solution with respect to robustness, speed and accuracy. The software uses a novel pattern recognition algorithm, which allows one to determine defocus and 2-fold astigmatism from a single diffractogram with a precision close to 1 Angstrom. Thereby it is possible to derive higher-order aberrations from a series of tilted diffractograms with an accuracy sufficient for 0.5 Angstrom microscopy. Investigations on the time stability of aberrations, and on the dependence of aberrations on certain operator actions are presented.

Concerning the determination of the microscope's information limit, we found that the traditional Young's fringe method is not suitable for an accurate measurement due to a couple of wellexplainable weaknesses. Instead, we introduce a new quantitative measurement method, which is based on the analysis of diffractograms taken under tilted illumination [1].

Concerning the camera modulation-transfer-function (MTF), it is remarkable that its measurement and incorporation into image simulations has not yet become a standard procedure. It is symptomatic that standard image simulation software even does not allow to include actually measured MTFs. We present here the case study of a focal series of $\mathrm{SrTiO}_{3}$, where the neglect of the MTF in image simulations leads to a contrast discrepancy between simulation and experiment, which is on the order of the frequently reported Stobbs-factor [2]. However, after incorporation of the measured MTF into image simulations, a contrast mismatch between simulation and experiment is no longer observed (see Figs. 1 and 2). In opposite, simulation and experiment are found to be perfectly consistent on an absolute contrast scale, indicating that a general contrast-mismatch phenomenon of huge size does not exist in HRTEM.

References

[1] J. Barthel and A. Thust, Phys. Rev. Lett. 101 (2008) 200801.

[2] M.J. Hytch and W.M. Stobbs, Ultramicroscopy 53 (1994) 191. 


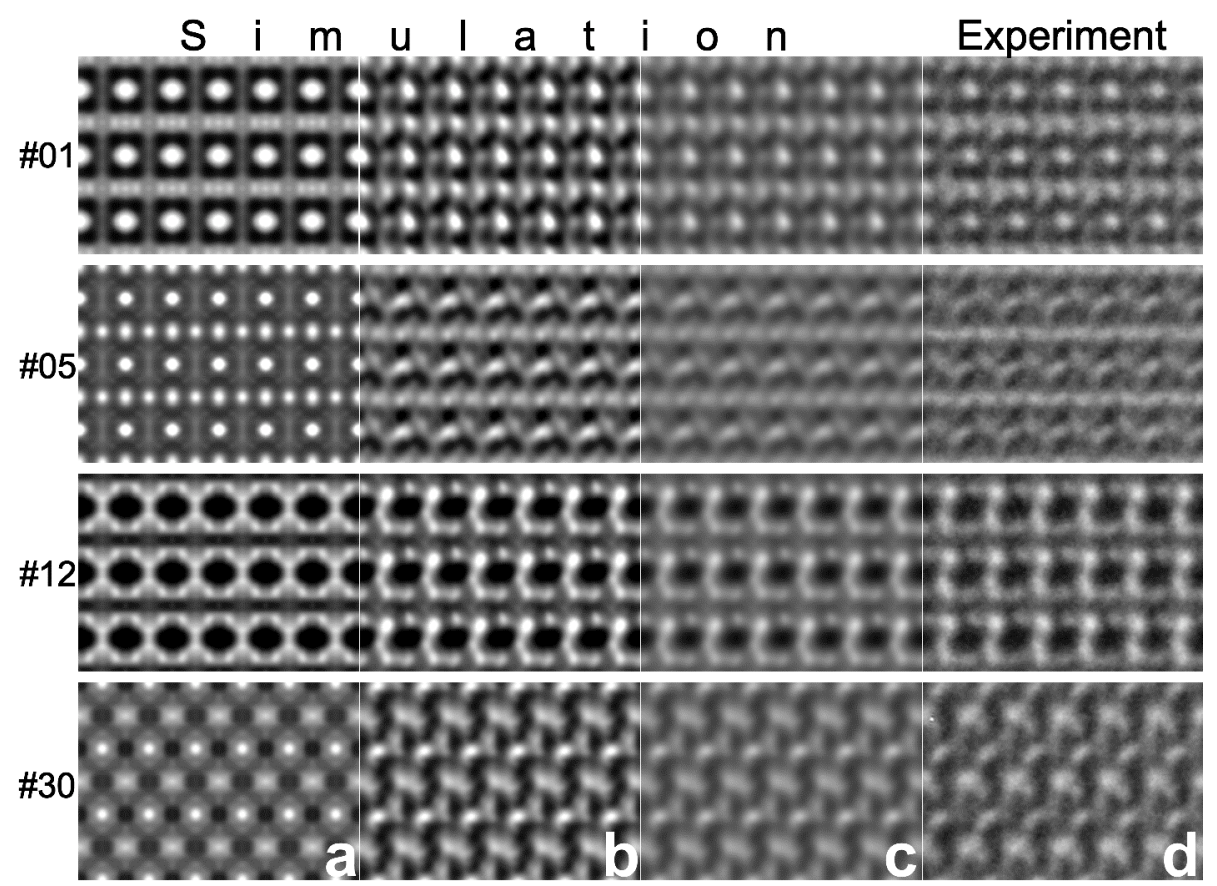

FIG. 1. HRTEM image patches comprising $3 \times 3 \mathrm{SrTiO}_{3}$ unit cells projected along the [110] zone axis. The patches $1,5,12$, and 30 , belong to different focal values and are shown exemplarily for a series of 30 images taken with a $\mathrm{C}_{\mathrm{S}}$-corrected FEI Titan 80-300 microscope equipped with a Gatan UltraScan 1000 camera. All patches are displayed on the same gray scale extending between the intensity values 0.35 (black) and 2.2 (white). (a) Ideal simulation for an object thickness of $2.8 \mathrm{~nm}$, (b) simulation including additionally residual lens aberrations, (c) simulation including residual aberrations and additionally the measured MTF, (d) experimentally recorded images.

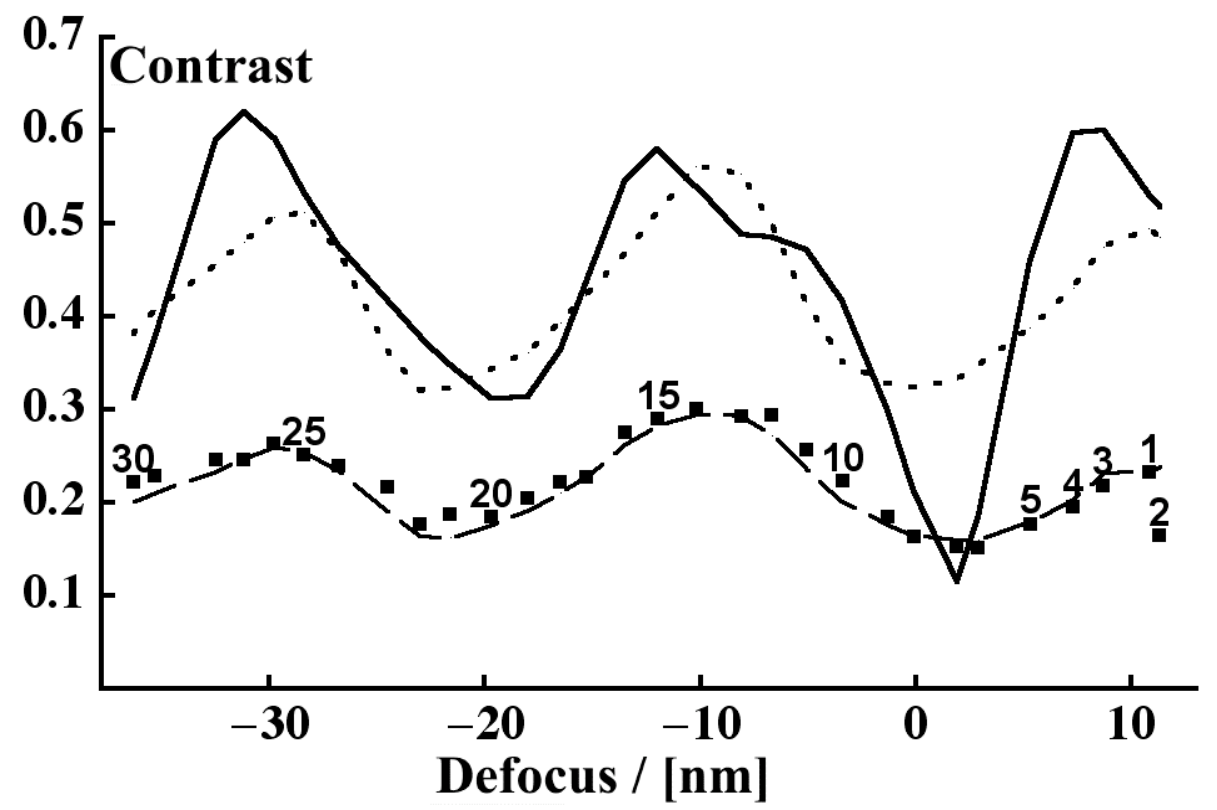

FIG. 2. Solid line: ideal image contrast simulated for an object thickness of $2.8 \mathrm{~nm}$ and for the focal values of the experimental $\mathrm{SrTiO}_{3}$ series. Dotted line: simulated contrast including residual lens aberrations. Dashed line: simulated contrast including residual aberrations and additionally the measured MTF. Squares: experimental contrast, image numbers within the focal series are indicated. 